\title{
Crystalline Structures of Some High Entropy Alloys Obtained by Neutron and X-Ray Diffraction
}

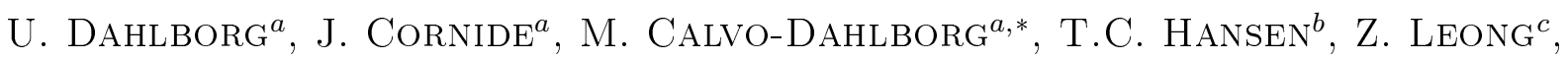
L. Asensio Dominguez ${ }^{c}$, S. Chambreland ${ }^{a}, \mathrm{~A}$ Cunliffe $^{a}$, R. Goodall ${ }^{c}$ And I. Todd ${ }^{c}$ ${ }^{a}$ GPM-UMR6634-CNRS, University of Rouen, BP12, 76801 Saint-Etienne-du-Rouvray, France

${ }^{b}$ Institute Laue-Langevin, BP 156, F-38042 Grenoble Cedex 9, France

${ }^{c}$ Department of Materials Science and Engineering, The University of Sheffield, Mappin Street, Sheffield S1 3JD, UK

\begin{abstract}
The understanding of the structure and the stability of high entropy alloys is still incomplete and the mechanisms behind the composition-property relationships are unclear. One reason is that few systematic and accurate determinations of their composition-dependent structure on atomic level have been made. In this paper some results on the structure obtained by X-ray and neutron diffraction of the CoCrFeNi alloy, to which $\mathrm{Pd}, \mathrm{Sn}$ and $\mathrm{Cu}$ have been added in different amounts, are reported. The investigations make it obvious that none of the alloys is completely homogeneous, as has earlier been suggested, and that they do not form a perfect solid solution.
\end{abstract}

DOI: 10.12693/APhysPolA.128.552

PACS: 61.05.cp, 61.05.fm, 61.66.Dk

\section{Introduction}

Metallic alloys consisting of five or more elements (socalled high entropy alloys, HEAs) have recently attracted large attention because of their good physical properties $[1,2]$ such as high hardness, outstanding wear resistance, good high-temperature strength and thermal stability but they also in some cases have surprising magnetic properties within certain composition ranges. However, the understanding of the structure and the stability of HEAs is still very incomplete and the mechanism behind the composition-property relationship is largely unclear. One reason is that few systematic and accurate determinations of the composition-dependent structure on the atomic level and of the physical properties of HEAs have been made. HEAs are usually equimolar or close to equimolar composition and form simple fcc or bcc phases in the solid phase. Furthermore, they are also assumed to form solid solutions, i.e. that the elements take random occupations on available lattice sites in a particular structure. However, additional intermetallic phases are also found in many cases. A large number of publications have been devoted to finding rules for whether an alloy after solidification forms a HEA or not [3-9].

The aim of this paper is, by using X-ray (XRD) and neutron diffraction (ND), to obtain more detailed information on the structure of one family of HEAs, namely those formed when adding a fifth element to the base $\mathrm{Co}_{25} \mathrm{Cr}_{25} \mathrm{Fe}_{25} \mathrm{Ni}_{25}$ (CCFN) alloy. Further investigations of the same alloys are presented in [10].

\footnotetext{
* corresponding author; e-mail:

monique.calvo-dahlborg@univ-rouen. fr
}

\section{Experimental details}

ND measurements have been performed on the D20 diffractometer at the Institute Laue-Langevin, Grenoble, France. The wavelength of the incident neutrons was $1.12 \AA$ with a maximum measured wave-vector transfer of $10.8 \AA^{-1}$ thus making the Rietveld refinement of the measured data meaningful. As neutrons are weakly interacting with matter the refinement gives a result valid for the entire irradiated volume. The experimental resolution was about $1 \%$ at $Q=3 \AA^{-1}$.

XRD measurements were for the structural investigation of the base CCFN alloy performed on different parts of the ingot. A Co $K_{\alpha}$ source $(\lambda=1.7889$ and $1.79825 \AA)$ was used which allowed a wave-vector range up to about $Q=6 \AA^{-1}$ to be covered. The resolution was about $0.1 \%$ at $Q=3 \AA^{-1}$. As the covered $Q$ range is small and only 4 diffraction peaks were observed in most cases, no refinement was performed but the indexing as well as the shape analysis of the measured diffraction peaks was carried out manually. The penetration depth of the X-rays in the CCFN alloy is of the order of $1 \mu \mathrm{m}$, and will thus give information of the structure only in a surface layer. As mentioned above ND on the contrary to XRD gives a measure of the bulk.

Ingots of $\mathrm{CCFN}-\mathrm{X}(\mathrm{X}=\mathrm{Cu}, \mathrm{Sn}, \mathrm{Pd})$ alloys were produced by arc melting of elements with purity higher than $99.9 \%$. In order for the alloys to be prepared in a way that makes them comparable to other studies each alloy was remelted at least 4 times to ensure good mixing of the elements. It should though be noted that it has been shown in numerous works that this procedure does not insure the alloy melt to be homogeneous ([11-13] and references therein). Cylindrical rods with a diameter of $3 \mathrm{~mm}$ were subsequently prepared by copper-mold suction casting. 


\section{Results and discussion}

\subsection{The structure of the base CCFN alloy}

The diffraction peak positions in the ND and XRD patterns from the CCFN alloy shown in Fig. 1 seem to correspond to a simple fcc structure. In order to minimize the influence of preferential grain orientations the sample in the ND measurements was rotating. Nevertheless, the variation of the peak intensities as well as their widths indicates that the samples have a poor grain statistics and contains a large amount of large grains. A closer inspection of the ND patterns suggests that the structure of the alloy may not entirely be characterized as being of a simple fcc type. Instead it indicates the existence of either a minority phase of similar structure or a fcc phase of a preferential ordering of the constituent atoms in the lattice resulting in a considerable strain. However, the resolution of the neutron diffraction measurement was not good enough to make any firm conclusions. The lattice constant obtained from a refinement by the FullProf computer code of the measured ND pattern in the $Q$ range $2<Q<10.5 \AA^{-1}$ yields the lattice constant equal to $3.580 \pm 0.002 \AA$.

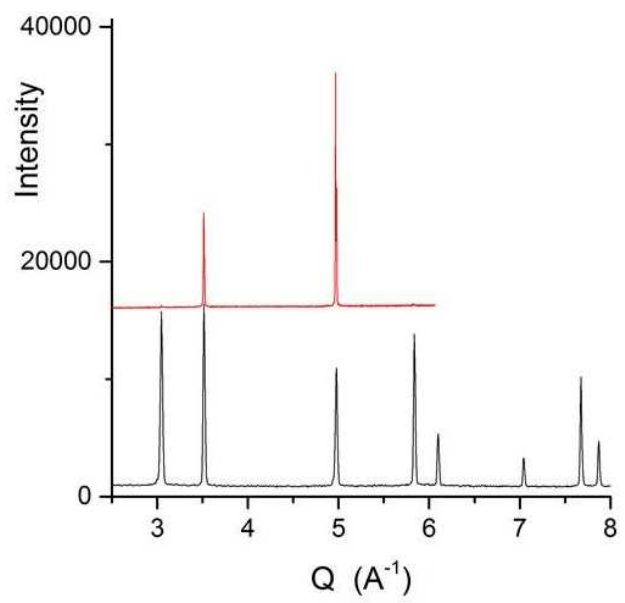

Fig. 1. Measured neutron (bottom) and X-ray (top) diffraction patterns from as-cast CCFN alloys.

For further investigation, XRD patterns were measured on six different pieces originating from different locations in the ingot. All measured diffraction patterns showed only diffraction peaks corresponding to a fcc structure. All diffraction peaks exhibit an asymmetry that could be qualitatively described by a fit of two Lorentz functions. This in turn can be interpreted as the existence of a minority phase also of fcc structure in the alloy. The uncertainty in the parameters defining this small extra peak is however too large to draw any quantitative conclusions. The lattice constants $d_{h k l}$ displayed in Fig. 2 have been derived from the maxima of the main (111), (200), (220) and (311) diffraction peak positions. The sample 1 to 6 correspond to XRD results while sample 7 corresponds to ND ones. The intensities and the widths of the different diffraction peaks in XRD measurements support the results from the ND measurements discussed above as they vary with $h k l$ in a way showing a distinct inhomogeneity of the ingot on the grain size level. The grain sizes were derived from the widths of the different as-fitted main $h k l$ peaks measured at the different positions and taking into account the experimental resolution. The obtained values varied from about 500 to larger than $5000 \AA$ demonstrating a large grain size and shape variation in accordance with the microscopy studies [10]. Furthermore, the lattice constants calculated from each separate peak, assuming a fcc lattice, vary, as can be seen in Fig. 2, slightly but significantly with the $h k l$ value. However, averaging over all samples and the positions of the XRD measured diffraction peaks yields a lattice constant deviating from the ND determined one with less than $0.0015 \AA$.

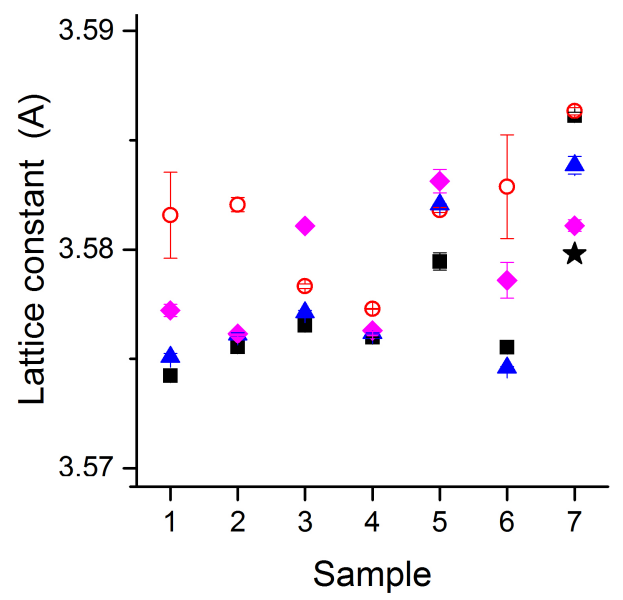

Fig. 2. Lattice constant, $a$, obtained from different diffraction peaks under the assumption that the lattice is fcc: $\square(111), \bigcirc(200), \Delta(220)$ and $\diamond(311)$. Samples 1 to 6 correspond to XRD obtained at different 1 positions in the ingot. Sample 7 corresponds to ND data. The star shows the lattice constant obtained from a refinement of all peaks in the ND, i.e. in a $Q$ range from 2.7 to $10.8 \AA^{-1}$.

Most of the earlier XRD measurements on the CCFN alloy have been interpreted as the alloy is single fcc phase and forms a perfect solid solution $([1,14,15]$ and references therein). However, the XRD diffraction peaks shown in [16] are clearly asymmetric in a way indicating the presence of two fcc phases in the alloy. The possible existence of a minority Cr-rich phase has furthermore been indicated in [17]. Not only details in the production process but also the production technique itself play an important role in the alloy phase composition. For example, after mechanical alloying, the alloy has been found to contain a minority bcc phase [18]. Furthermore, lattice constants, spanning a range from 3.55 to $3.59 \AA$, in many cases determined from one measured XRD diffraction peak only, have been reported. All results presented and/or recalled above clearly demonstrate the ambiguity in earlier reported works. 


\subsection{The effects of adding a fifth element to the CCFN alloy}

The structural changes occurring by adding three different kinds of atoms ( $\mathrm{Sn}, \mathrm{Cu}$ and $\mathrm{Pd}$ ) in equimolar amounts to the CCFN alloy were studied by XRD. The measured patterns, all measured from as-cast samples, are shown in Fig. 3. At first glance, the addition of Pd does not change the fcc structure apart from a lattice expansion. On the other hand, when $\mathrm{Sn}$ and $\mathrm{Cu}$ are added, the alloys retain their initial fcc structures but some new phases appear. All diffraction peaks are wider than the instrumental resolution and also in some cases asymmetric which indicates that a deeper analysis of the measured diffraction patterns has to be performed.

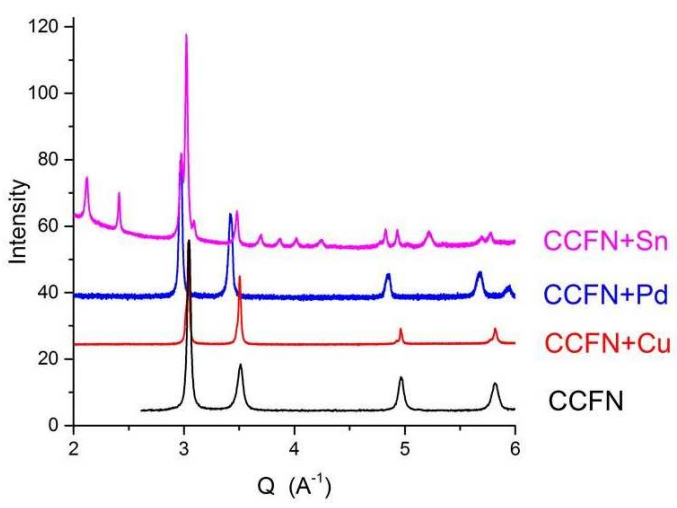

Fig. 3. Measured XRD patterns from the CCFN alloy and from alloys to which has been added equimolar amounts of $\mathrm{Cu}, \mathrm{Pd}$ and $\mathrm{Sn}$.

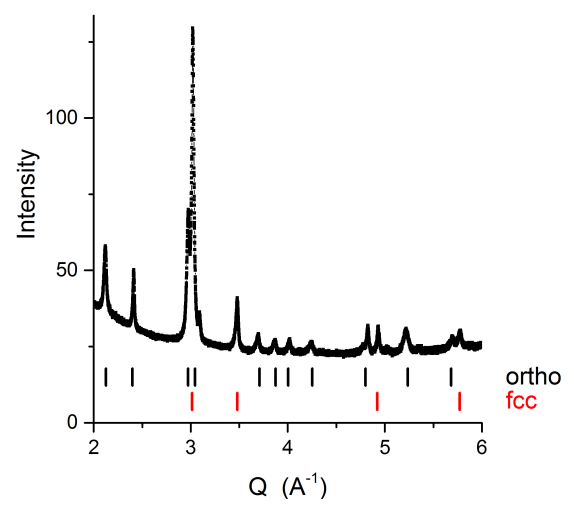

Fig. 4. Measured XRD pattern from the CCFN-Sn $\mathrm{Sn}_{1.0}$ alloy. The vertical bars show the diffraction peak positions according to a fcc lattice with $a=3.611 \AA$ and an orthorhombic lattice with $a=7.15 \AA, b=5.24 \AA$ and $c=8.27 \AA$.

In the measured XRD pattern for $\mathrm{CCFN}-\mathrm{Sn}_{1.0}$ (Fig. 4), all diffraction peaks can be indexed assuming one fcc and one orthorhombic phase. The lattice constant of the fcc phase is $a=3.611 \pm 0.006 \AA$ while the o-phase is defined with $a=7.15 \pm 0.01, b=5.24 \pm 0.01$ and $c=8.27 \pm 0.01 \AA$ (Table). The fcc structure of the initial CCFN alloy $(a=3.580)$ has thus expanded slightly by addition of Sn. The size of the o-lattice coincides almost exactly with the one given for $\mathrm{Ni}_{3} \mathrm{Sn}_{2}$ in the JCPDS data sheets. CCFN-Sn $n_{x}$ alloys with $x$ in the range 0 to 0.5 have been studied by Liu [18]. An extrapolation to $x=1.0$ yields a lattice constant of about $3.615 \AA$ in good agreement with the present work. SEM studies of the alloys for $x=0.5[18]$ and $x=1.0[10]$ have shown that the microstructures of the ingots were dendritic with Sn- and Ni-rich interdendritic regions.

TABLE

Structural characteristics of the investigated alloys obtained by X-ray diffraction on as-cast alloys.

\begin{tabular}{|c|c|c|c|}
\hline $\begin{array}{c}\text { CCFN } \\
\text { addition }\end{array}$ & \multicolumn{3}{|c|}{ Phases } \\
\hline none & & $\begin{array}{c}\text { fcc; } \\
a=3.580 \pm 0.002\end{array}$ & \\
\hline $\mathrm{Cu}_{0.5}$ & $\begin{array}{c}\text { fcc; } \\
a=3.574 \pm 0.005\end{array}$ & & \\
\hline $\mathrm{Cu}_{1.0}$ & $\begin{array}{c}\text { fcc; } \\
a=3.575 \pm 0.008\end{array}$ & $\begin{array}{c}\text { fcc; } \\
a=3.583 \pm 0.004\end{array}$ & $\begin{array}{c}\text { fcc; } \\
a=3.606 \pm 0.006\end{array}$ \\
\hline $\mathrm{Cu}_{1.5}$ & & $\begin{array}{c}\text { fcc: } \\
a=3.583 \pm 0.004\end{array}$ & $\begin{array}{c}\text { fcc; } \\
a=3.608 \pm 0.004\end{array}$ \\
\hline $\mathrm{Sn}_{1.0}$ & $\begin{array}{c}\text { fcc: } \\
a=3.611 \pm 0.006\end{array}$ & $\begin{array}{c}\text { Orthorhombic } \\
a=7.15 \pm 0.01 \\
b=5.24 \pm 0.01 \\
c=8.27 \pm 0.01\end{array}$ & \\
\hline $\mathrm{Pd}_{0.5}$ & $\begin{array}{c}\text { fcc; } \\
a=3.631 \pm 0.004\end{array}$ & $\begin{array}{c}\text { fcc; } \\
a=3.653 \pm 0.007\end{array}$ & \\
\hline $\mathrm{Pd}_{1.0}$ & $\begin{array}{c}\text { fcc; } \\
a=3.662 \pm 0.005\end{array}$ & $\begin{array}{c}\text { fcc; } \\
a=3.678 \pm 0.005\end{array}$ & \\
\hline $\mathrm{Pd}_{1.5}$ & $\begin{array}{c}\text { fcc; } \\
a=3.705 \pm 0.007\end{array}$ & $\begin{array}{c}\text { fcc; } \\
a=3.722 \pm 0.004\end{array}$ & \\
\hline
\end{tabular}

The measured diffraction patterns for $\mathrm{CCFN}-\mathrm{Cu}_{x}$ shown in Fig. 5 indicate an overall fcc structure. However, all the peaks seem to be split. In order to investigate this further measurements were performed also on $\mathrm{CCFN}-\mathrm{Cu}_{x}$ alloys with $x=0.5$, and 1.5 . The measured patterns for the three $\mathrm{Cu}$ concentrations shown in Fig. 5 indicate that the size of the splitting depends on $\mathrm{Cu}$ concentration. In order to illustrate this further the (111) and (220) diffraction peaks are displayed separately in Fig. 6. It can be seen that for $x=0.5$ both peaks consist of one main peak and possible one small at smaller $\mathrm{Q}$ values. For $x=1.0$, the equimolar composition, three components and for $x=1.5$ two to three can be identified, all belonging to fcc structures. The derived lattice constants are given in Table. For $x=0.5$ the results indicate that the alloy to a large extent retains the structure of the pure CCFN alloy. However, all peaks for this $\mathrm{Cu}$ content show a small asymmetry suggesting a presence of a small amount of a second phase. The asymmetry is though considered to be too small to be quantified. The amount of CCFN phase is according to Fig. 6 decreasing with increase of $\mathrm{Cu}$ content of the 
alloy. Instead another fcc phase with slightly larger unit cell is growing together with a third one with an even larger cell dimension (see Table). It can be noted that the lattice constant of the third phase obtained from fits of three Lorentz functions to all diffraction peaks is in fair agreement with the one of pure $\mathrm{Cu}(a=3.615 \AA)$. Taking into account the instrumental resolution the width values of all measured peaks yield alloy grain sizes in the range 300 to $500 \AA$.

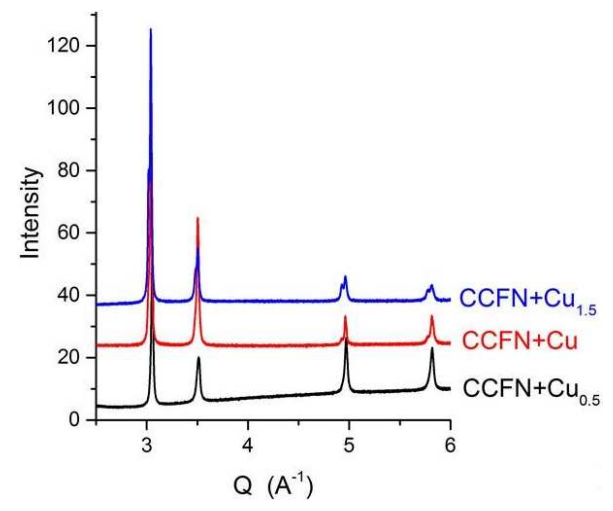

Fig. 5. Measured XRD patterns from $\mathrm{CCFN}-\mathrm{Cu}_{x}$ alloys at three $\mathrm{Cu}$ concentrations $(x=0.5,1.0,1.5)$, 1.0 being equimolar.

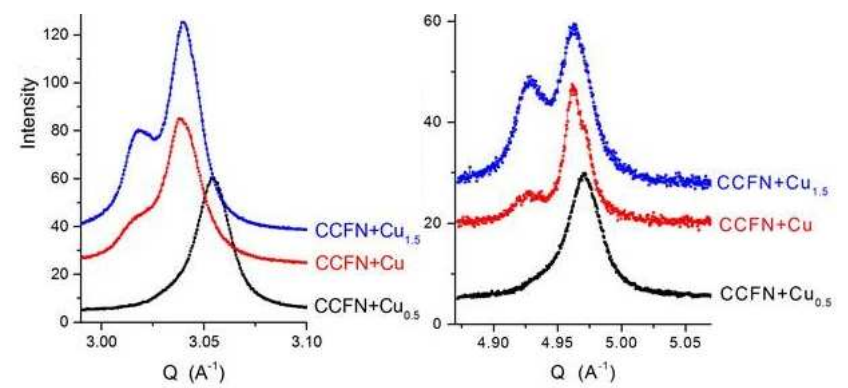

Fig. 6. The (111) and (220) diffraction peaks measured from $\mathrm{CCFN}-\mathrm{Cux}$ alloys at three $\mathrm{Cu}$ concentrations $(x=$ $0.5,1.0,1.5), 1.0$ being equimolar.

The structure and physical properties of $\mathrm{CCFN}-\mathrm{Cu}_{1.0}$ equimolar alloy have been investigated in [15] where it is claimed that the alloy is single-phase. However, a second minority fcc phase was identified in [14] together with the main fcc phase with $a=3.579 \AA$. The $\mathrm{CCFN}-\mathrm{Cu}_{1.0}$ alloy was shown to have a dendritic structure, the interdendritic region being enriched in $\mathrm{Cu}$ and associated with the minority fcc phase [19]. These results agree closely with the results presented above. Furthermore, an alloy produced by mechanical alloying was found after $15 \mathrm{~h}$ of milling to contain a minority bcc phase together with a main fcc phase [20]. In alloy films only a fcc structure was detected at ambient temperature [21]. The different results are most probably due to different sample preparation techniques and alloy grain size distributions.
It can thus be concluded that the $\mathrm{CCFN}-\mathrm{Cu}$ alloys are multi-phase and that the base CCFN alloy is present in a decreasing amount with increasing $\mathrm{Cu}$ content of the alloy.

The addition of $\mathrm{Pd}$ to the CCFN alloy does not have an effect on the general structure (Fig. 3), the only obvious effect being an expansion of the fcc lattice. However, the peak shapes are irregular and vary significantly with $\mathrm{Pd}$ concentration. This is clearly seen in Fig. 7, which shows the (311) peak for CCFN-Pd $\mathrm{Pd}_{x}(x=0,0.5,1.0$ and 1.5), 1.0 being equimolar. All peaks, except the one for $\mathrm{CCFN}$, are considerably wider than the instrumental resolution and can well be described by a sum of two Lorentzian functions which indicates the presence of two coexisting fcc structures. The derived grain sizes for both sets of diffraction peaks all lie in the range 300 to $600 \AA$.
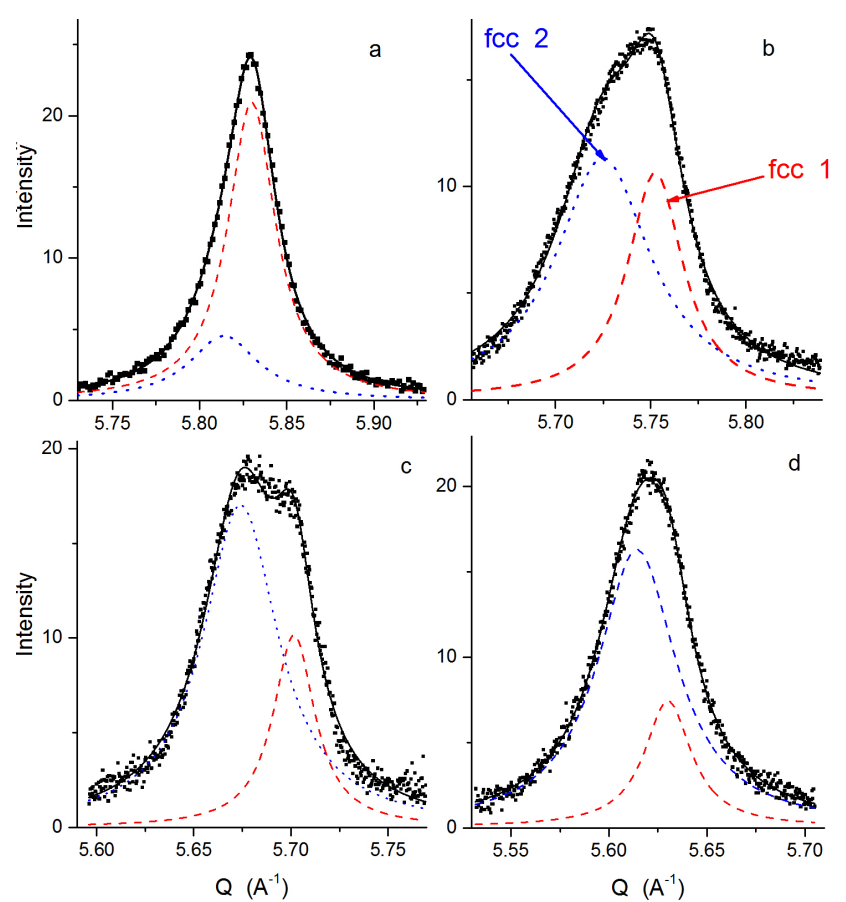

Fig. 7. The (311) diffraction peak in the measured XRD patterns from CCFN-Pd ${ }_{x}$ alloys for (a) $x=0.0$, (b) $x=0.5$, (c) $x=1.0$ (equimolar) and (d) $x=1.5$ $(--)$ and (...) correspond to the two components, fcc-1 and fcc- 2 , respectively.

Furthermore, for pure CCFN the measured peak can also be described by a sum of two Lorentzians but in this case it might indicate the presence of a frustrated structure as the peak positions are very close and the peak width is only slightly larger than the resolution.

The lattice distances derived from fits for one CCFN$\mathrm{Pd}_{0.5}$, three $\mathrm{CCFN}-\mathrm{Pd}_{1.0}$ and two $\mathrm{CCFN}-\mathrm{Pd}_{1.5}$ ingots are shown in Fig. 8 where also the results from [22], that are in fair agreement with the present results, are included. The agreement between the different data sets in the present work is quite satisfactory and average values are given in Table. 


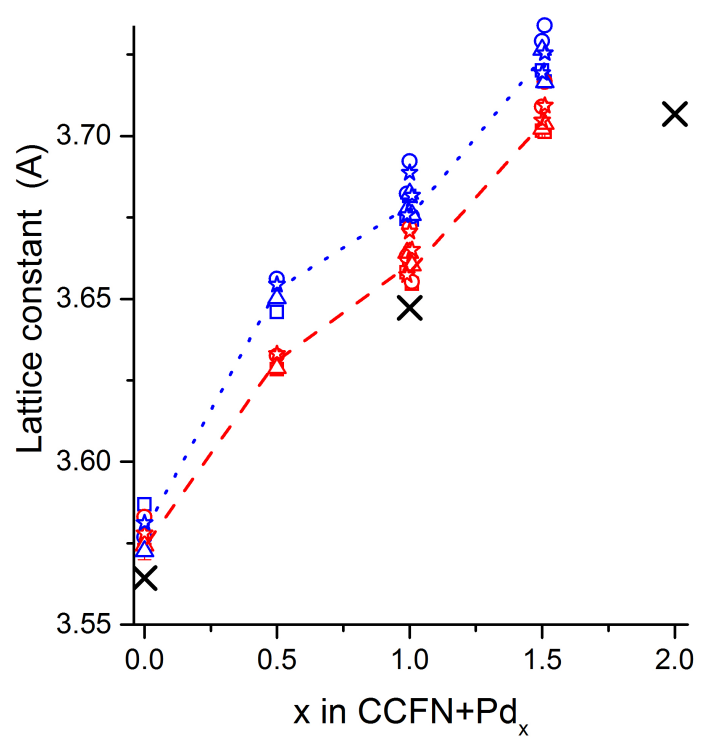

Fig. 8. Lattice constants corresponding to the two intensity components in Fig. 7, fcc-1 values are connected by the dashed curve and fcc- 2 values by the dotted one, calculated under the assumption that the lattice is fcc. $\square$ (111) diffraction peak, $\bigcirc(200), \Delta(220)$ and $\diamond(311)$. The crosses are values measured by Lucas et al. [22].

\section{Conclusions}

X-ray and neutron diffraction measurements have shown that the structure of high entropy alloys based on $\mathrm{CoCrFeNi}$ in as-cast conditions are not as simple as earlier been reported.

Specifically for the CoCrFeNi alloy itself it has been found that the grain orientation is highly anisotropic in all investigated samples. From the spread of the lattice parameters by XRD at different positions in the ingot and from ND the element distribution on available lattice positions is most probably not random which is in accordance with composition dependent fluctuations observed in investigations by atom probe tomography. Furthermore, the as-cast alloy is not single-phase but consists of at least two phases, at least one being of fcc structure.

The $\mathrm{CoCrFeNi}-\mathrm{Cu}_{x}(x=0.5,1.0,1.5)$ alloys are found to be multi-phase and the base $\mathrm{CoCrFeNi}$ structure still exists at small $\mathrm{Cu}$ concentrations in the alloy. At large $\mathrm{Cu}$ concentrations the alloy is two-phase, one phase having a structure close to elemental $\mathrm{Cu}$.

The $\mathrm{CoCrFeNi}-\mathrm{Pd}_{x}(x=0.5,1.0,1.5)$ and the $\mathrm{CoCrFeNi}-\mathrm{Sn}_{1.0}$ alloys are multiphase contrary to what has earlier been reported.

\section{Acknowledgments}

This work has been performed in the framework of the EU-FP7 project AccMet.

\section{References}

[1] Y. Zhang, T.T. Zuo, Z. Tang, M.C. Gao, K.A. Dahmen, P.K. Liaw, Z.P. Lu, Prog. Mater. Sci. 61, 1 (2014).

[2] M.H. Tsai, J.W. Yeh, Mater. Res. Lett. 2, 107 (2014).

[3] A. Cunliffe, J. Plummer, I. Figueroa, I. Todd, Intermetallics 23, 204 (2012).

[4] R. Raghavan, K.C. Hari Kumar, B.S. Murty, J. Alloys Comp. 544, 152 (2012).

[5] S. Guo, Q. Hu, C. Ng, C.T. Liu, Intermetallics 41, 96 (2013).

[6] F. Otto, Y. Yang, H. Bei, E.P. George, Acta Mater 61, 2628 (2013).

[7] F. Zhang, C. Zhang, S.L. Chen, J. Zhu, W.S. Cao, U.R. Kattner, CALPHAD 45, 1 (2014).

[8] A.K. Singh, N. Kumar, A. Dwivedi, A. Subramanian, Intermetallics 53, 112 (2014).

[9] M.G. Poletti, L. Battezzati, Acta Mater. 75, 297 (2014).

[10] J. Cornide, M. Calvo-Dahlborg, S. Chambreland, L. Asensio Dominguez, Z. Leong, U. Dahlborg, A. Cunliffe, R. Goodall, I. Todd, Acta Phys. Pol. A 128, 557 (2015).

[11] U. Dahlborg, M. Calvo-Dahlborg, Mater. Sci. Eng. A 283, 153 (2000).

[12] V. Manov, P. Popel, E. Brook-Levinson, V. Molokanov, M. Calvo-Dahlborg, U. Dahlborg, V. Sidorov, L. Son, Yu. Tarakanove, Mater. Sci. Eng. A 304-306, 54 (2001).

[13] M. Calvo-Dahlborg, U. Dahlborg, J.M. Ruppert, J. Non-Cryst. Solids 357, 798 (2011).

[14] Z.F. Wu, H. Bei, F. Otto, G.M. Pharr, E.P. George, Intermetallics 46, 131 (2014).

[15] S. Guo, C. Ng, Z. Wang, C.T. Liu, J. Alloys Comp. 583, 410 (2014).

[16] M.S. Lucas, G.B. Wilks, L. Mauger, J.A. Muňoz, O.N. Senkov, E. Michel, J. Horwath, S.L. Semiatin, M.B. Stone, D.L. Abernathy, E. Karapetrova, Appl. Phys. Lett. 100, 251907 (2012).

[17] A.K. Singh, A. Subramaniam, J. Alloys Comp. 587 , 113 (2014).

[18] L. Liu, J.B. Zhu, C. Zhang, J.C. Li, Q. Jiang, Mater. Sci. Eng. A 548, 64 (2012).

[19] Yu-Jui Hsu, Wen-Chi Chiang, Jiann-Kuo Wu, Mater. Chem. Phys. 92, 112 (2005).

[20] S. Praveen, B.S. Murthy, R.S. Kottada, Mater. Sci. Eng. A 534, 83 (2012).

[21] Z.F. Wu, X.D. Wang, Q.P. Cao, G.H. Zhao, J.X. Li, D.X. Zhang, J.J. Zhu, J.Z. Jiang, J. Alloys Comp. 609, 137 (2014).

[22] M.S. Lucas, L. Mauger, J.A. Muňoz, Y. Xiao, A.O. Sheets, S.L. Semiatin, J. Horwath, Z. Turgut, J. Appl. Phys. 109, 07E307 (2011). 